\title{
Spectroscopic evaluation of vibrational temperature and electron density in reduced pressure radio frequency nitrogen plasma
}

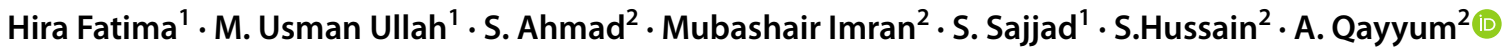

Received: 9 March 2021 / Accepted: 10 May 2021

Published online: 17 May 2021

(c) The Author(s) 2021

OPEN

\begin{abstract}
The optical emission spectroscopy technique is used to determine the vibrational temperature of the second positive band system, $N_{2}\left(C, v^{\prime}-B, v^{\prime \prime}\right)$ in the wavelength range $367.1-380.5 \mathrm{~nm}$ by using the line-ratio and Boltzmann plot methods. The electron temperature is evaluated from the intensity ratio of the selected molecular bands corresponding to $N_{2}^{+}\left(B, v-X, v^{\prime}, 391.44 \mathrm{~nm}\right)$, and, $N_{2}\left(C, v^{\prime}-B, v^{\prime \prime}, 375.4 \mathrm{~nm}\right)$ transitions, respectively. The selected bands have a different threshold of excitation energies and thus serve as a sensitive indicator of the electron energy distribution function (EEDF). The electron density has been determined from the intensity ratio of the molecular transitions corresponding to $N_{2}^{+}\left(B, v-X, v^{\prime}, 391.44 \mathrm{~nm}\right)$, and, $N_{2}\left(C, v^{\prime}-B, v^{\prime \prime}, 380.5 \mathrm{~nm}\right)$ for different levels of pressure and radio frequency power. The results show that the vibrational temperature decreases with increasing nitrogen fill pressure and radio frequency power. However, the electron temperature increases with radio frequency power and reduces with fill pressure. The electron density increases both with nitrogen fill pressure and radio frequency power that attributes to the effective collisional transfer of energy producing electron impact ionization. Plasma parameters show a significant dependence on discharge conditions and can be fine-tuned for specific surface treatments.
\end{abstract}

\section{Article Highlights}

- Spectrum analysis of RF-driven nitrogen plasma for varying discharge conditions

- Evaluation of vibrational temperature using line-ratio and Boltzmann plot methods
- Comparison of vibrational temperatures for line-ratio and Boltzmann plot methods

- Evaluation of electron temperature and density using the intensity-ratio of bands

- Correlation of temperature and density with varying fill pressure and RF power

Keywords Radio frequency-driven plasma - Vibrational temperature - Electron impact excitation · Line-ratio method . Boltzmann plot method

\section{Introduction}

Radio frequency plasma-based surface treatment such as etching, sputtering, and thin-film deposition is the emerging research field aimed at diverse industrial applications
[1-3]. Therefore, studies on radiofrequency (RF) discharges are essential to select the plasma conditions for specific surface modifications to have potential applications in the field of material and electronic sciences. It is affectionate to produce low-density, low-temperature plasma

A. Qayyum, abdul.qaui@gmail.com | 1 Institute of Space Technology (IST), Islamabad, Pakistan. ${ }^{2}$ Pakistan Tokamak Plasma Research Institute (PTPRI), Islamabad, Pakistan.

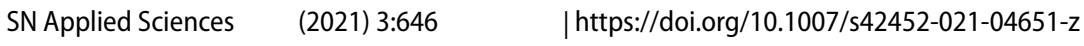


in RF discharge for high-tech industrial applications. RF sources are someway more advanced than DC sources regardless of some common properties. They demonstrate well-controlled plasma kinetics and have more ability to excitation/ionization. RF plasma sources are generally subdivided into two types depending on their energy coupling mechanisms. These types are inductively coupled plasma (ICP) and capacitively coupled plasma (CCP). Both configurations can use electrodes conductive or nonconductive, built within the tube of low-pressure gas or outside of it. Commonly RF plasma source with a frequency of $13.56 \mathrm{MHz}$ in the capacitively coupled configuration is preferred for surface treatments $[4,5]$.

Plasma spectroscopy has a wide range of applications in the field of plasma analysis and parametric optimization. The most convenient and commonly used method for the diagnostic of reactive plasma is optical emission spectroscopy (OES) because it does not perturb the plasma, like other diagnostic tools such as Langmuir probes. The plasma particles go through electron impact excitation and subsequent de-excitation emitting light of a specific wavelength. These radiations provide the details of the plasma species and their energies, making OES in the visible range an attractive method for plasma analysis with an easily manageable experimental setup [6-8]. The line radiations from plasma take in some broadening in their energy levels owing to different broadening mechanisms including natural broadening, pressure broadening, Doppler broadening, and Stark broadening, etc. Consequent broadening in the wavelength of spectral lines depends on the plasma environments [9-11]. Alternatively, the intensity of the spectral line is sensitive to the shape of the electron energy distribution function (EEDF), electron temperature, and electron number density $\left(n_{e}\right)$, fundamental parameters characterizing the discharge conditions.

Molecular spectroscopy deals with three basic modes of molecular excitation named rotational, vibrational, and electronic, and subsequent de-excitation. However, here the main focus is on the electron impact excitation of the vibrational and the electronic states of the molecule to determine the vibrational and the electron temperatures. In a vibrational mode of excitation, the electrons gain energy from the applied electric field and transfer to nitrogen molecules through the collisional process. As a result, the nitrogen molecules jump to the higher vibrational levels without changing the electronic level and subsequently de-excited by emitting photons of characteristic wavelengths in the infrared region. The identification of the spectral lines/bands and quantification of their respective intensities give information on the energy and density of the charged particles, the population density of the excited states, and plasma composition. The knowledge of vibrational temperature is essential to understand the physical and chemical properties of $\mathrm{N}_{2}$ discharge. Qianghua et al. [12] studied the vibrational temperature of second positive system of $\mathrm{N}_{2}$ for varying values of RF power at a fixed fill pressure of 0.26 mbar. Levaton et al. [13] investigated the effect of gas flow rate, fill pressure and discharge current on the emission intensity of the second positive system of $\mathrm{N}_{2}$, and vibrational temperature. They found that vibrational temperature has a strong dependence on the discharge conditions. The reactive nature of nitrogen plasma makes it very suitable for surface modification and deposition. Plasma nitriding is the most useful method for surface treatments and has potential industrial applications [14].

The paper reports the vibrational temperature obtained from the relative intensity of the bands of the second positive system $N_{2}\left(C^{3} \Pi_{u} \rightarrow B^{3} \Pi_{u}\right)$ with sequence $(\Delta v=-2)$ for different values of RF power and nitrogen pressure at a fixed frequency of $13.56 \mathrm{MHz}$. The information of the electron temperature and number density is also mandatory to understand the physical properties of the electric discharge. To characterize the large group of the electron's energy distribution, the intensity of the $\mathrm{N}_{2}$-bands having a large difference in the excitation threshold energies has been used to determine the electron temperature. Therefore, the intensity-ratio of the emission bands belonging to the first negative system $N_{2}^{+}\left(B^{2} \sum_{u} \rightarrow X^{2} \Sigma_{g}^{+}\right)$at $391.44 \mathrm{~nm}$, and the second positive system $N_{2}\left(C^{3} \Pi_{u} \rightarrow B^{3} \Pi_{u}\right)$ at $375.4 \mathrm{~nm}$, and $380.5 \mathrm{~nm}$ is analyzed for electron temperature and density respectively.

\section{Experimental details}

For optical characterization of capacitively coupled RFgenerated nitrogen plasma between two disk-like electrodes, spectroscopic analysis is performed for different levels of RF power and nitrogen fill pressure. The vacuum chamber is a cylindrical shape made of Pyrex glass that provides the best possible visibility for the discharge dynamics and eliminates the need for extra optical windows for light collection to make plasma observations. The height and diameter of the chamber are $28-\mathrm{cm}$ and $16-\mathrm{cm}$. The plasma chamber includes upper and bottom parallel disc-like circular brass electrodes assembly with a diameter of $5-\mathrm{cm}$, and the distance between electrodes is $2.5-\mathrm{cm}$. The upper electrode serves as the power electrode, and the bottom electrode is grounded, serves as the cathode for the glow discharge. Digital clamp multimeter (UT-201) measures discharge current. The voltage between discharge electrodes is monitored by True RMS Multimeter (FLUKE-175). Digital storage oscilloscope (Tektronix TDS 2014C) attached to Pearson's current 
transformer has been used to monitor the shape of the discharge current. To avoid impurities not being added to the plasma during discharge the electrodes are insulated with ceramic material except the front side facing the plasma discharges. Figure 1 shows the schematic diagram of the experimental setup along with the optical components of the diagnostic system.

The chamber has four vacuum-tight multi-purposes ports. The optical fiber coupled with Ocean HR 2000 + high-resolution spectrometer is used to collect and record the optical emission spectrum for wavelength characterization in the range is from 248 to $465 \mathrm{~nm}$. It provides a line of sight measurement of the light emission from the plasma glow region. A rotary vane pump is coupled to one of the ports and has been used to get pressure down in the plasma chamber to working level. Another port connects Bayard-Alpert Pirani Gauge FRG720 coupled with a pressure-displaying unit to monitor the working pressure inside the plasma chamber during the plasma generation and characterization. The third port is used to enter the nitrogen gas in the vacuum chamber in a controlled way. A matching network is an essential part of the plasma generation system and helps to couple maximum RF power to plasma discharge by minimizing the power reflection. Its function is basically to equalize the output impedance of the generator to the load of the varying discharge that changes with the discharge conditions. Therefore, it seems necessary to add an auto-matching network between the RF generator and dc-electrode plasma discharge for the efficient coupling of $13.56 \mathrm{MHz}$ RF power that generates the plasma between two disk-like electrodes housed in the glass chamber. The RF generator is manufactured by Plasma Technology Ltd for Lab experiments.

\section{Spectrum analysis and evaluation of plasma parameters}

The corona model is quite a satisfactory approach to determine the electron temperature $\left(T_{e}\right)$ and number density $\left(n_{e}\right)$ in the low temperature and low-density non-LTE plasmas. Herein the electron temperature, ion temperature, and temperature of neutral particles follow the relation $\left(T_{e}>T_{i}>T_{n}\right)$. For the nitrogen, the main part of the observed spectra belongs to the second positive system, which results from the transition $N_{2}\left(C^{3} \Pi_{u} \rightarrow B^{3} \Pi_{u}\right)$ where $C^{3} \Pi_{u}$ is the upper state and having higher energy than $B^{3} \Pi_{u}$ (lower state) [15]. The main reaction pathway involving the electron impact excitation from the ground state $N_{2}(X, v=0)$ to the excited state $N_{2}\left(C, v^{\prime}\right)$ is as follows [15-17]

$e+N_{2}(X, v=0) \rightarrow e+N_{2}\left(C, v^{\prime}\right) \quad\left(E_{\mathrm{th}}=11.1 \mathrm{eV}\right)$

Subsequently, the radiative de-excitation is given by

$N_{2}\left(C, v^{\prime}\right) \rightarrow N_{2}\left(B_{,}^{\prime \prime}\right)+h v$

For the specific $\left(C, v^{\prime}\right) \rightarrow\left(B, v^{\prime \prime}\right)$ transition, the emission intensity of the second positive system is stated as $[6,18]$.

$I_{C B}\left(v^{\prime}, v^{\prime \prime}\right)=C(\lambda) n_{n_{2}} n_{e} X_{X}^{C}\left(T_{e}\right) \frac{A_{C B}\left(v^{\prime}, v^{\prime \prime}\right)}{\sum_{v^{\prime \prime}} A_{C B}\left(v^{\prime}, v^{\prime \prime}\right)}$

where $n_{n_{2}}$ represent the number density of $N_{2}(X, v=0)$, the $\mathrm{n}_{\mathrm{e}}$ is the number density of electrons, $X_{X}^{C}\left(T_{e}\right)$ is the excitation rate coefficient for electron impact excitation, $A_{C B}$ is the Einstein coefficient and $C(\lambda)$ is the spectral response of spectrometer at specific wavelength $\lambda$ [18]. The other major part of nitrogen spectra is the first negative system mentioned early. The positive ion of nitrogen $N_{2}^{+}\left(B, v^{\prime}\right)$
Fig. 1 Schematic diagram of RF-driven plasma source with RF Generator, auto-matching network, and optical spectrometer

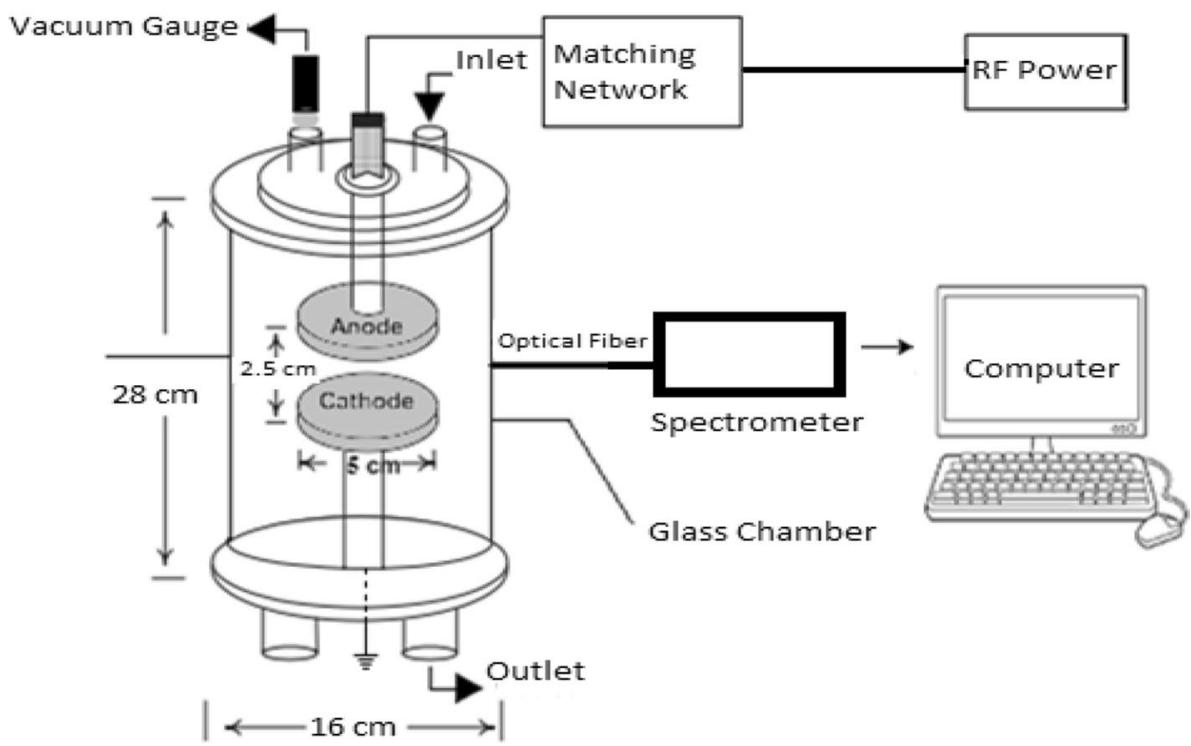


can also be produced through electron impact ionization either by direct or step-wise following the channels is given by $[19,20]$

$e+N_{2}(X, v=0) \rightarrow N_{2}^{+}\left(X, v^{\prime}\right)+2 e \quad\left(E_{\mathrm{th}}>15.6 \mathrm{eV}\right)$

$e+N_{2}^{+}(X, v=0) \rightarrow N_{2}^{+}\left(B, v^{\prime}\right)+e \quad\left(E_{\mathrm{th}}>3.3 \mathrm{eV}\right)$

The pathway of radiative de-excitation of $N_{2}^{+}\left(B, v^{\prime}\right)$ state is described as [21]

$N_{2}^{+}\left(B, v^{\prime}\right) \rightarrow N_{2}^{+}(X, v=0)+h v$

The resulting emission intensity of $N_{2}^{+}\left(B^{2} \Sigma_{u} \rightarrow X^{2} \Sigma_{g}^{+}\right)$ band is stated as [18]

$I_{B X}^{+}\left(v^{\prime}, v^{\prime \prime}\right)=C(\lambda)\left[\left(n_{n_{2}} n_{e} X_{X}^{B^{+}}\left(T_{e}\right)\right)+\left(n_{e}^{2} X_{X}^{B^{+}}\left(T_{e}\right)\right)\right] \frac{A_{B X}^{+}\left(v^{\prime}, v^{\prime \prime}\right)}{\sum_{v^{\prime \prime}} A_{B X}^{+}\left(v^{\prime}, v^{\prime \prime}\right)}$

The emission spectra of the first negative system $N_{2}^{+}\left(B, v^{\prime \prime}=0-X, v^{\prime}=0\right)$ are observed at $391.44 \mathrm{~nm}$ while the spectra of the second positive system have different bands of vibrational transitions $(\Delta v)$, that are $\Delta v=1$, $\Delta v=0, \Delta v=-1, \Delta v=-2, \Delta v=-3$, and $\Delta v=-4$ as shown in Fig. 2 [22]. The sequential intensity of vibrational bands of the second positive system $N_{2}\left(C^{3} \Pi_{u} \rightarrow B^{3} \Pi_{g}\right)$ is related to the population density of the respective state as below $[23,29]$.

$I_{v^{\prime} v^{\prime \prime}}=C(\lambda) \frac{\left[N, v^{\prime}\right] A_{v^{\prime} v^{\prime \prime}}}{\lambda}$

where $I_{v^{\prime}} v^{\prime \prime}$ is the emission intensity between two vibrational levels, $v^{\prime}$ and $v^{\prime \prime}$ while $v^{\prime}$ is the upper vibrational level and $v^{\prime \prime}$ is the lower vibrational level, $C(\lambda)$ is for the spectral response of spectrometer, $A_{v^{\prime} v^{\prime \prime}}$ is the transition probability or Einstein coefficient and $\lambda$ is the respective wavelength. If we evaluate the emission intensity of each band of the specific wavelength and know the value of each corresponding constant, it is very easy to find the value of the respective excited state population $N, v^{\prime}$ [1]. The labeling and intensity distributions of emission bands/lines in the experimental spectrums for the first negative system (a)

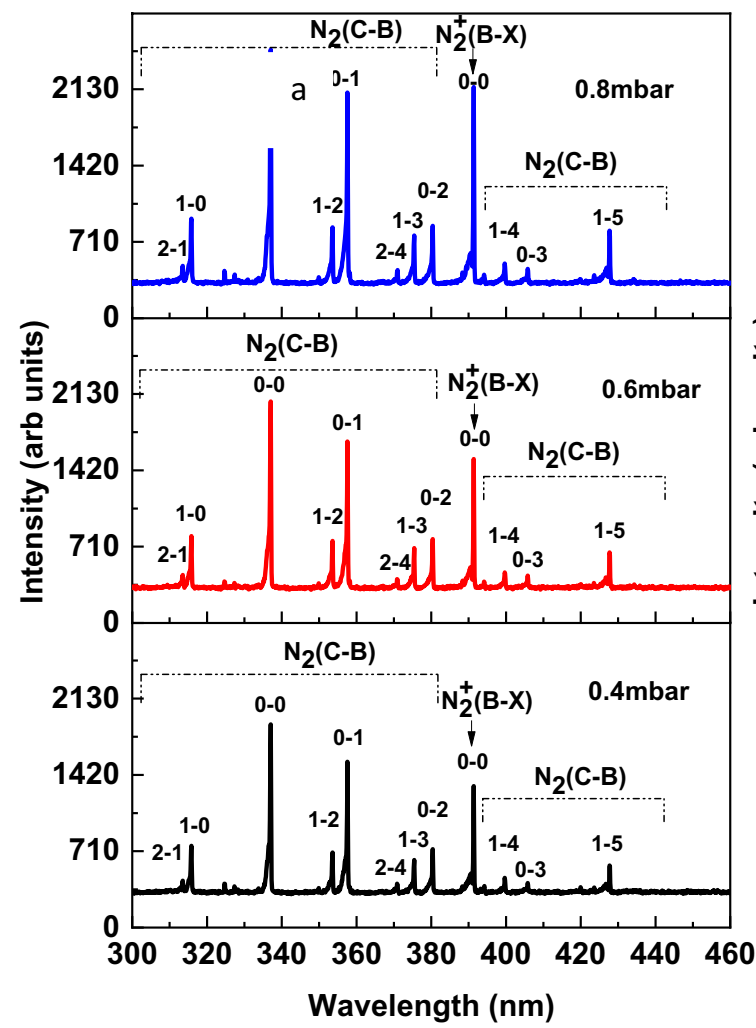

(b)

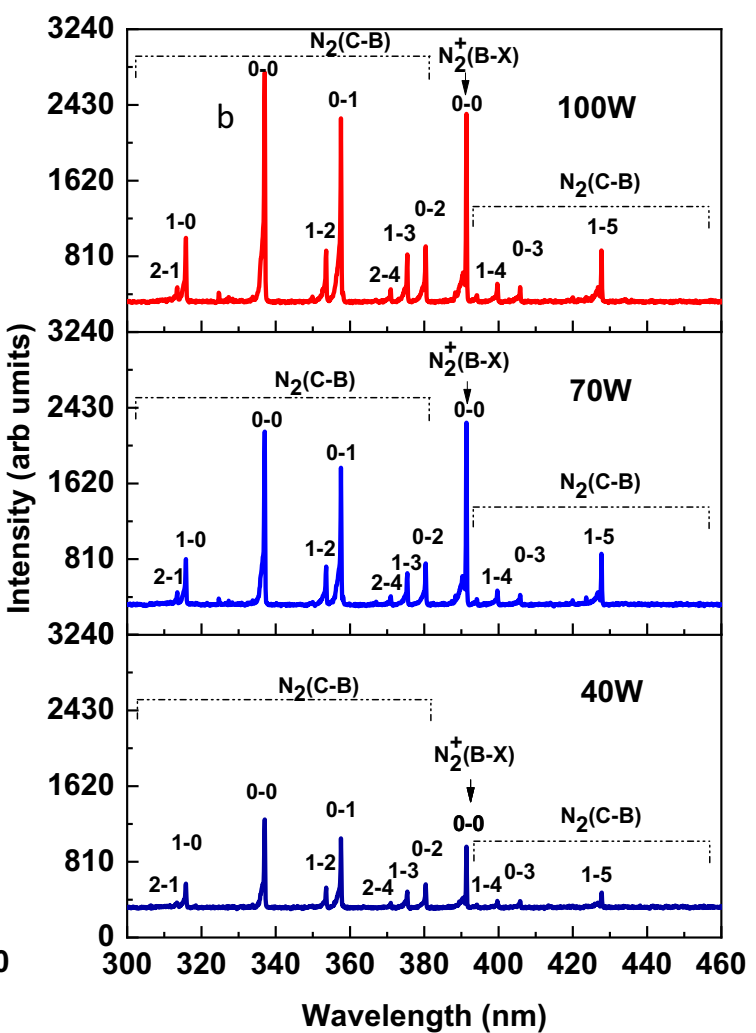

Fig. 2 Emission spectra of RF $\mathrm{N}_{2}$-discharge. a for different nitrogen pressures $0.4 \mathrm{mbar} 0.6 \mathrm{mbar}$ and $0.8 \mathrm{mbar}$ at fixed power $80 \mathrm{~W}$. $\mathbf{b}$ for different RF power $40 \mathrm{~W}, 70 \mathrm{~W}$, and $100 \mathrm{~W}$ at fixed nitrogen fill pressure of $1.0 \mathrm{mbar}$ 
$N_{2}^{+}(B-X)$ and the second positive system $N_{2}(C-B)$ are presented in Fig. 2.

Figure 3 shows the emission of second positive band system for vibrational transitions $\Delta v=-2$ observed at different nitrogen pressure and RF power. The band for $\Delta v=-2$ at different pressure and power is shown in Fig. 3. In this band, we can see four emission line $N_{2}\left(C, v^{\prime}=3-B, v^{\prime \prime}=5,367.1 \mathrm{~nm}\right), N_{2}\left(v^{\prime}=2-B, v^{\prime \prime}=4\right.$, $371 \mathrm{~nm}), N_{2}\left(v^{\prime}=1-B, v^{\prime \prime}=3,375.4 \mathrm{~nm}\right)$ and $N_{2}\left(v^{\prime}=0-B, v^{\prime \prime}=2,380.5 \mathrm{~nm}\right)$. The spectra at different nitrogen fill pressures varying from 0.1 to $1 \mathrm{mbar}$ at constant RF power $80 \mathrm{~W}$, and frequency $13.56 \mathrm{MHz}$ are presented in Fig. 3. The spectral lines at 0.4 mbar, 0.6 mbar, and 0.8 mbar are shown in Fig. 3a. Alternatively, we recorded the intensities of these spectral lines at different values of RF power that is from 10 to $100 \mathrm{~W}$ with the difference of $10 \mathrm{~W}$ at constant pressure $1 \mathrm{mbar}$ and frequency 13.56 MHz. Some observed intensities of these spectral lines at different power that is $40 \mathrm{~W}, 70 \mathrm{~W}$ and $100 \mathrm{~W}$ are shown in Fig. $3 \mathrm{~b}$. The vibrational temperature $T_{v}$ is estimated by using two different methods, one is the Boltzmann plot method while the other one is the line-ratio method. Here it is assumed that the plasma particles are in a steady state so excited states follow the Boltzmann distribution. For the Boltzmann plot method, the population densities of the upper excited state of $N_{2}(C-B)$ band that is $N_{2}\left(C, v^{\prime}\right)$ versus corresponding vibrational energies $\mathrm{G}\left(v^{\prime}\right)$ is plotted to determine the slope.

The populations' densities have been inferred from the measured intensities of selected bands using relation 3 . The values of wavelength, transition probabilities, and vibrational term values are given in Table 1. The population densities the upper state $N_{2}\left(C, v^{\prime}\right)$ are plotted versus the $\mathrm{G}\left(v^{\prime}\right)$ for different values of nitrogen fill pressure and RF power. Some of the plots for selected values of discharge

Table 1 Spectroscopic constants used in the calculations [24-26]

\begin{tabular}{lllll}
\hline$\lambda(\mathrm{nm})$ & $\mathrm{A}_{v^{\prime} v^{\prime \prime}}\left(\mathrm{Sec}^{-1}\right)$ & $\mathrm{v}^{\prime}$ & $\mathrm{v}^{\prime \prime}$ & $\mathrm{G}\left(\mathrm{v}^{\prime}\right)\left(\mathrm{cm}^{-1}\right)$ \\
\hline 380.49 & 0.134 & 0 & 2 & 1016 \\
375.54 & 0.185 & 1 & 3 & 2987 \\
371.05 & 0.151 & 2 & 4 & 4764 \\
367.19 & 0.0868 & 3 & 5 & 6103 \\
\hline
\end{tabular}

(a)

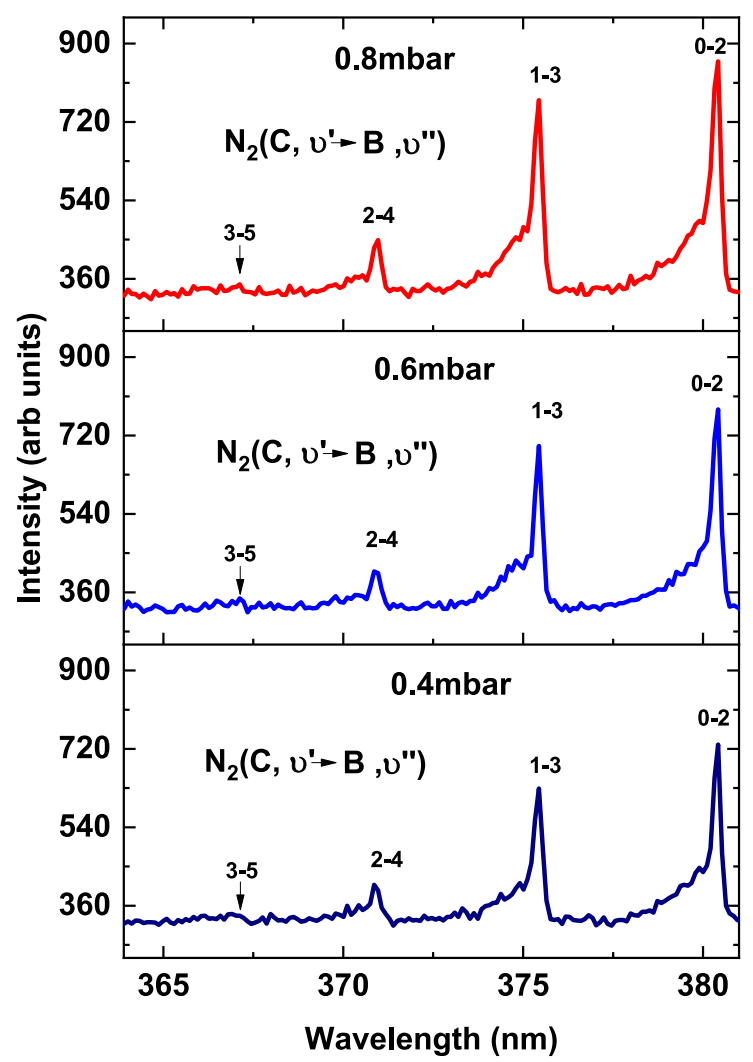

(b)

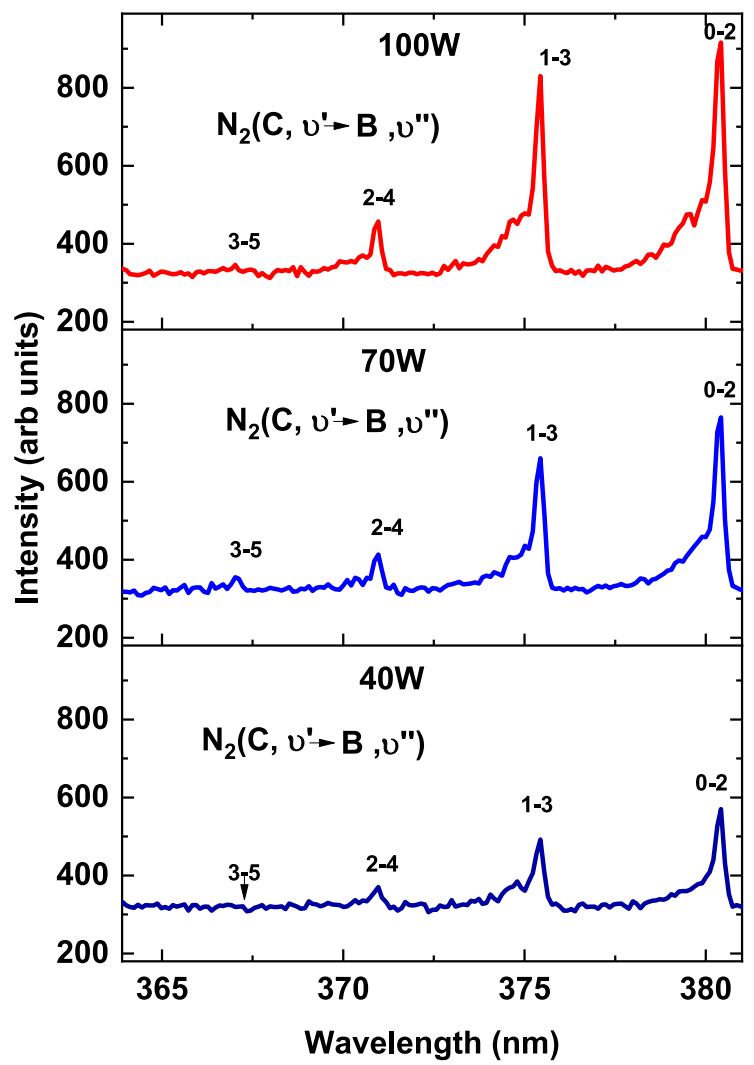

Fig. 3 Emission spectra of $\mathrm{N}_{2}$ second positive band $\mathrm{N}_{2}(\mathrm{C}-\mathrm{B})$ at wavelengths $367.19 \mathrm{~nm}, 371.05 \mathrm{~nm}, 375.54 \mathrm{~nm}$, and $380.49 \mathrm{~nm}$ a for different pressure $0.4,0.6$, and $0.4 \mathrm{mbar}$ at RF power $80 \mathrm{~W}$. $\mathbf{b}$ for different RF power $40 \mathrm{~W}, 70 \mathrm{~W}$ and $100 \mathrm{~W}$ at nitrogen fill pressure of $1.0 \mathrm{mbar}$ 
pressure are shown in Fig. 3a and for different RF power are shown in Fig. $3 b$.

$\mathrm{G}\left(v^{\prime}\right)=\frac{E_{v^{\prime}}}{h c}=\omega_{e}\left(v^{\prime}+\frac{1}{2}\right)-\omega_{e} x_{e}\left(v^{\prime}+\frac{1}{2}\right)^{2} \ldots$

Here, $\mathrm{c}$ is the speed of light that is $3 \times 10^{8} \mathrm{~ms}^{-1}$, $\mathrm{h}$ is Planck's constant $E_{v^{\prime}}$ is the vibrational energy of upper excited state $\mathrm{N}_{2}\left(\mathrm{C}, v^{\prime}\right), \omega_{e}$ and $\omega_{e} x_{e}$ are Herzberg's vibrational constant [12]. Figure 4 shows that the Boltzmann plot results in an almost straight line suggesting the EEDF behaves as Maxwellian. Accordingly, the vibrational temperature can be readily evaluated from the slope of the Boltzmann plots on a semi-log scale. During the analysis of the population densities, the role of the metastable states has been neglected owing to their small concentration in the discharge. Another method simple in vogue for the calculation of vibrational temperature is the line-ratio method.

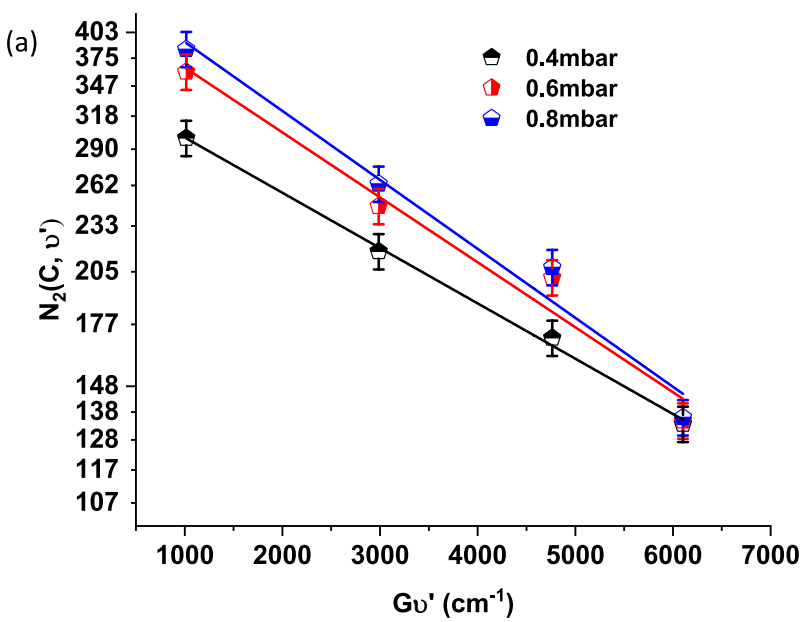

(b)

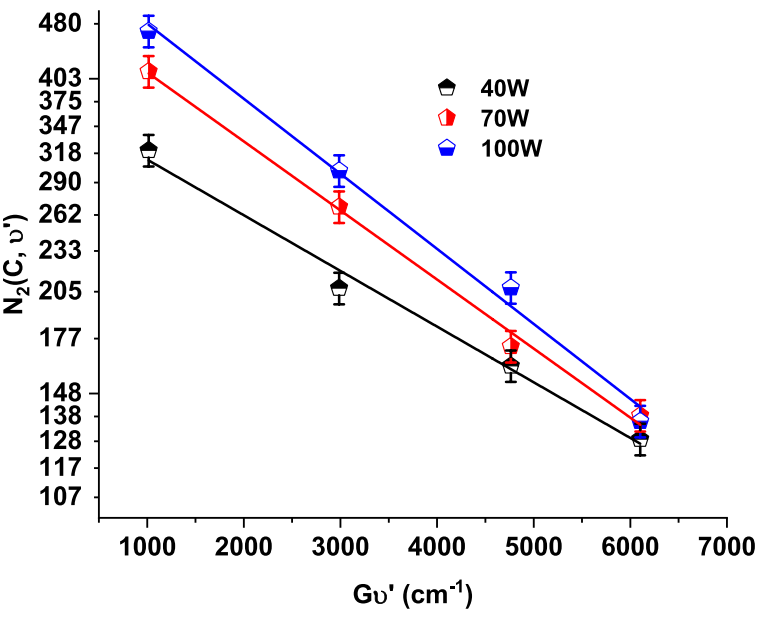

Fig. 4 The Boltzmann plot for the determination of vibrational temperature at $\Delta v=-2$, where $\mathrm{N}_{2}\left(\mathrm{C}, v^{\prime}\right)$ is the population density of $\mathrm{C}$ state. $\mathbf{a}$ at different gas pressure for constant RF power $80 \mathrm{~W}$, b for different RF power at constant nitrogen fill pressure of $1.0 \mathrm{mbar}$
Because in low-density plasma, the dominant pathway of the excitation is only electron impact and the effect of cascading is not considered important for populating of $\mathrm{N}_{2}\left(C, v^{\prime}\right)$ state [15]. So it seems convenient and reliable to use the line-ratio method for the calculation of vibrational temperature [27]. Equation 3 can be written as,

$I_{v^{\prime} v^{\prime \prime}}=C(\lambda) A_{v^{\prime} v^{\prime \prime}} \frac{2 \pi c}{\lambda} e^{-\frac{E_{v^{\prime}}}{k_{B} T_{v}}}$

The intensity ratio of two bands belonging to the second positive system $N_{2}\left(C^{3} \Pi_{u} \rightarrow B^{3} \Pi_{u}\right)$ at the wavelength $371 \mathrm{~nm}$ and $380.5 \mathrm{~nm}$ are used to determine the vibrational temperature via a line-ratio method. The relation for intensity ratio can be easily derived using Eq. 5 while incorporating the values of known spectroscopic constants given in Table 1 .

$\frac{I_{1 v^{\prime} v^{\prime \prime}}(371.1 \mathrm{~nm})}{I_{2 v^{\prime} v^{\prime \prime}}(380.5 \mathrm{~nm})}=1.126 e^{-\frac{0.465}{T_{v}}}$

OES is a widely used diagnostic tool for measuring the electron temperature $\left(T_{e}\right)$ and electron number density $\left(n_{e}\right)$ in industrial plasmas. There are different methods to determine the $T_{e}$ and $n_{e}$ from the intensity distribution of the measured spectrum. Here the line-ratio method that uses the intensities of two selected emission bands of RF nitrogen plasma is analyzed for electron temperature. For line-ratio method, it is assumed that the electrons' velocity distribution is Maxwellian, and the excitation from the ground states to upper states follows the Boltzmann distribution [28-30]. However, in low-pressure RF discharges, there is some deviation from the Maxwellian behavior due to inefficient collisional transfer from electrons to other plasma species to equilibrate their temperatures. Despite this fact, the evaluation of electron temperature from the line intensities with different excitation energy thresholds represents an energy scan of the electrons. Because in low electron density plasmas, the corona model is generally applicable that assumes the population of the upper levels mainly by the direct electron impact excitation without any multi-stage excitation and the de-excitation radiative. Consequently, the electron temperature is sensitive to the relative intensity of two spectral lines having different excitation energy thresholds. Therefore, the line-ratio method gives reasonably accurate results in the case of low electron density, non-LTE plasmas with some deviation from Maxwellian $\operatorname{EEDF}[18,30]$. Explicitly the final relation comes as:

$k_{B} T_{e}=\frac{E_{2}-E_{1}}{\ln \frac{l_{1} \lambda_{1} g_{2} A_{2}}{I_{2} \lambda_{2} g_{1} A_{2}}}$

Here $I_{1}$ and the $I_{2}$ is the intensity of the selected bands from the second positive and the first positive systems 
at wavelengths $375.4 \mathrm{~nm}$, and $391.44 \mathrm{~nm}$, respectively, $\lambda$ is the wavelength, $E$ is excitation energy, $g$ is statistical weight, and $A$ is transition probability. We measured the electron temperature and number density at different values of nitrogen pressure ranging from $0.1 \mathrm{mbar}$ to $1 \mathrm{mbar}$ at fixed power $80 \mathrm{~W}$, and different values of RF power ranging from 10 to $100 \mathrm{~W}$ at fixed pressure $1 \mathrm{mbar}$. To determine the electron number density, we used the ratio of two emission bands at $391.44 \mathrm{~nm}$ from $N_{2}^{+}(B-X)$ and the other one is at $380.5 \mathrm{~nm}$ from $N_{2}(C-B)[29,30]$.

$n_{e}=\left(\frac{I_{391.44 n m}}{I_{380.50 n m}}-0.0108\right) \times \frac{10^{17} m^{-3}}{1.048}$

\section{Result and discussion}

In RF discharges, plasma species get excited to the higher energy states owing to electron impact excitation and then de-excite to a lower energy state by emitting a photon of characteristic energy and wavelength. Using $\mathrm{OES}$, it is very affectionate to characterize the particular emission lines/bands present in the emission spectrum and consequently the energy of charged particles the plasma composition. Hence, OES provides detailed information on the excitation and ionization processes involved in the optical emission of different molecules, atoms, and their ions. The intensities of emission lines may be related to the population densities of respective excited states safely if the concentration of metastable states in the discharge is negligible. If the transitions have a different threshold of excitation energies, they encompass different energy groups of electrons for electron impact excitation. As a result, any change in the emission intensity-ratio suggests a variation in the EEDF, and corresponding vibrational and electron temperatures. However, the analysis becomes more difficult in the presence of a large number of metastable states. Figure 2 shows the emission lines of the band head of the first negative system $N_{2}^{+}(B-X)$ at $391.44 \mathrm{~nm}$ and the band head of the second positive system $N_{2}(C-B)$ at $380.5 \mathrm{~nm}$ for different nitrogen fill pressure and RF power. The intensity of spectral bands increases with increasing RF power and similarly for nitrogen fill pressure. However, the increase in the intensity of the first negative system $N_{2}^{+}(B-X)$ at $391.44 \mathrm{~nm}$ is more apparent relative to the second positive system $\mathrm{N}_{2}(C-B)$ at $380.5 \mathrm{~nm}$ with RF power. The discharge parameters actuality has a great influence on the shape of the $E E D F$, and consequently on the population densities of excited state such as $N_{2}^{+}\left(B, v^{\prime}\right)$ and $\mathrm{N}_{2}\left(C, v^{\prime}\right)$. The excitation energy of the state $N_{2}^{+}\left(B, v^{\prime}\right)$ is $18.7 \mathrm{eV}$ whereas the excitation energy of $N_{2}\left(C, v^{\prime}\right)$ is $11.1 \mathrm{eV}$. It shows that the threshold excitation energy of the state $N_{2}^{+}\left(B, v^{\prime}\right)$ is greater than the excitation energy of $N_{2}\left(C, v^{\prime}\right)$. Therefore, the population of the $N_{2}^{+}\left(B, v^{\prime}\right)$ state is more sensitive to the high energy electron compared with the $\mathrm{N}_{2}\left(C, v^{\prime}\right)$ state. All these factors affect the determination process of vibrational temperature, electron temperature, and electron number density. Figure 3 shows the dependence of emission spectra of $\mathrm{N}_{2}$ second positive band system $N_{2}(C,-B)$ at wavelengths $367.19 \mathrm{~nm}, 371.05 \mathrm{~nm}$, $365.4 \mathrm{~nm}$, and $380.49 \mathrm{~nm}$ for different nitrogen pressure and RF power. The vibrational temperature is based on the emission intensity analysis of the second positive system $N_{2}\left(C, v^{\prime}-B, v^{\prime \prime}\right)$. Figure 4 shows the Boltzmann plot at different nitrogen fill pressure RF power and presents the relative population of the upper state $N_{2}\left(C, v^{\prime}\right)$ against the vibrational energy term on a semi-log scale. Because the intensity of the band resulted from the transition between two vibrational levels with sequence $\Delta v=-2$ can be measured and all the constants are known, it is possible to obtain the relative population of the upper states from the respective intensities. The slope of the Boltzmann plot gives the vibrational temperature at different nitrogen pressure, and RF power when other corresponding discharge parameters kept constant.

Figure 5 a shows the variation of vibrational temperature with nitrogen fill pressure. The values of nitrogen pressure are varied from $0.1 \mathrm{mbar}$ to $1 \mathrm{mbar}$ in regular steps by fixing RF power at the level of $80 \mathrm{~W}$ at a frequency of $13.56 \mathrm{MHz}$. The vibrational temperature has been obtained using the line-ratio and Boltzmann plot methods. The values of vibrational temperature with fill pressure vary from 0.78 to $0.39 \mathrm{eV}$ in decreasing order for Boltzmann plot method, and from 0.99 to $0.51 \mathrm{eV}$ in decreasing order for line ratio method. Figure $5 b$ demonstrates the effect of increasing power from 10 to $100 \mathrm{~W}$ at a pressure of $1 \mathrm{mbar}$ on the vibrational temperature. The values of vibrational temperature show the decreasing trend for the both Boltzmann plot, and for the line-ratio methods. This fact may be attributed to the collisional relaxation of nitrogen molecule at higher gas pressure, and fast quenching which causes the reduction of vibrational temperature. The values vary from 0.56 to $0.36 \mathrm{eV}$ for the Boltzmann plot method, and 0.88-0.53 eV for the line-ratio method, respectively. The value of $T_{v}$ measured from the line-ratio method is somewhat higher than the value of $T_{v}$ estimated from the Boltzmann plot method. It is because the line-ratio method involves only the intensity contribution of two spectral lines (smaller group of the electrons) while the Boltzmann plot method involves the contribution from several spectral lines. Therefore, the Boltzmann plot method represents more shares of 

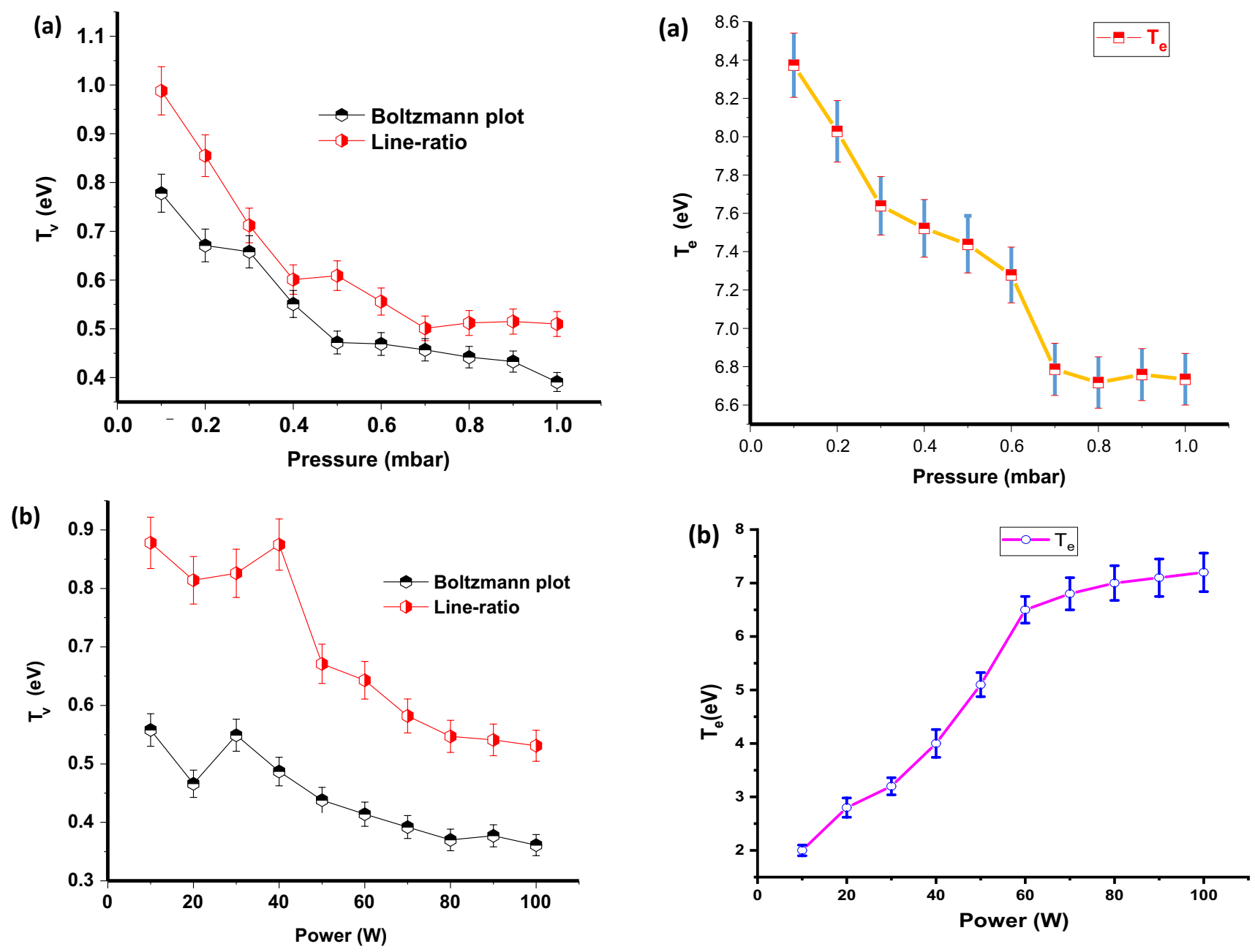

Fig. 5 Variations of vibrational temperature with a nitrogen fill pressure, b RF power

the electrons present in the EEDF compared with the line-ratio method which represents a smaller group of electrons, and measures the vibrational temperature more accurately.

Figure 6a demonstrates the relationship between nitrogen fill pressure and the corresponding electron temperature $T_{e}$ at the given value of the RF power. The electron temperature is determined by the line-ratio method that uses the relative intensity ratio of the molecular bands corresponding to $N_{2}^{+}\left(B, v-X, v^{\prime}, 391.44 \mathrm{~nm}\right)$, and, $\mathrm{N}_{2}\left(C, v^{\prime}-B, v^{\prime \prime}, 375.4 \mathrm{~nm}\right)$, transitions, and known spectroscopic constants. The results show that the $T_{e}$ continuously decreases with increasing nitrogen fill pressure. This outcome can be explained as follows: When the working pressure of nitrogen increases, it shorten the mean free path and increases the collisional frequency among the discharge particles. As a result of an effective collisional transfer of energy, the electrons are cool down, causing a reduction in electron temperature. Figure $6 \mathrm{~b}$ shows the

Fig. 6 Electron temperature measurements in $\mathrm{N}_{2}$ RF-generated discharge. $\mathbf{a}$ The variation of $T_{e}$ with nitrogen fills pressure. $\mathbf{b}$ The variation of $T_{e}$ with RF power

increasing trend of electron temperature versus the RF power. This is because the electrons receive more energy at a higher value of the RF field at higher input power and transfer to the plasma species through the collisional process. The collisional process increases the population density of the emitting states and consequently the intensity of the spectral lines. The electron temperature is related to the population density of the excited-states involved in the optical emission process, subject to the multi-stage excitation/de-excitation is negligible.

The high-energy electrons having energies above the excitation thresholds contribute to the excitation/ionization of the plasma species via inelastic collisions. The excitation rate coefficient is the function of the electron energy and the electron number density. Therefore, with increasing RF powers, the inelastic collisions between high-energy electrons and plasma particles increase producing more, ion-electron pairs, and excited plasma 
species. As a result of the dissipation of electron energy, the electrons are relatively cooled and reduce the tail of the EEDF. However, both the electron temperature and the electron density increase with RF power due to the continuous absorption of RF power compensating the energy loss of the electrons. Figure 7a shows the variation of the electron density with varying nitrogen fill pressure at constant RF power. As nitrogen fills pressure increases, it causes an increase in the rate of collisions among the electrons and the other plasma species. Consequently, the collisional transfer of energy by way of the electron impact ionization results in charge multiplication. Therefore, as a result of charge multiplication, the electron density increases while the electron temperature decreases due to the energy loss of the electrons by the collisional processes. Figure $7 \mathrm{~b}$ shows the variation of the electron

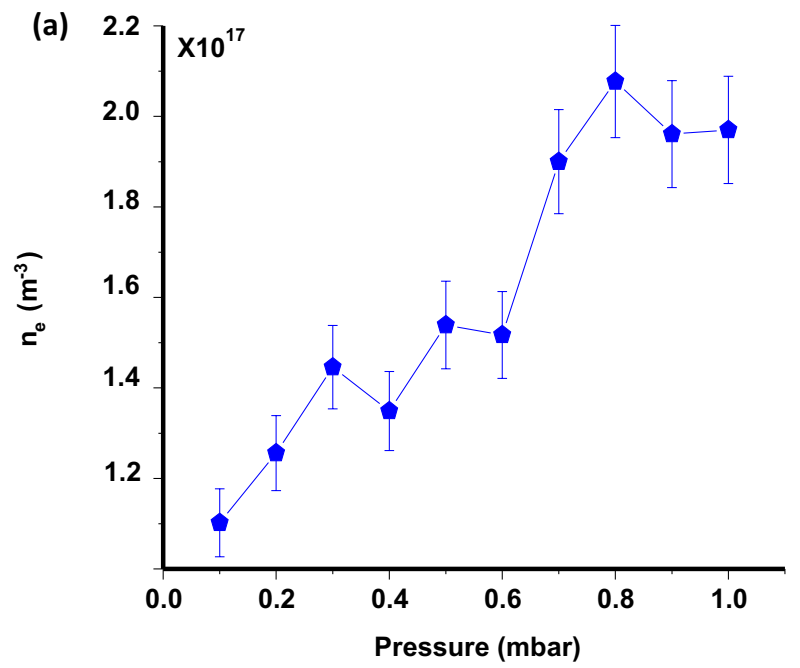

(b)

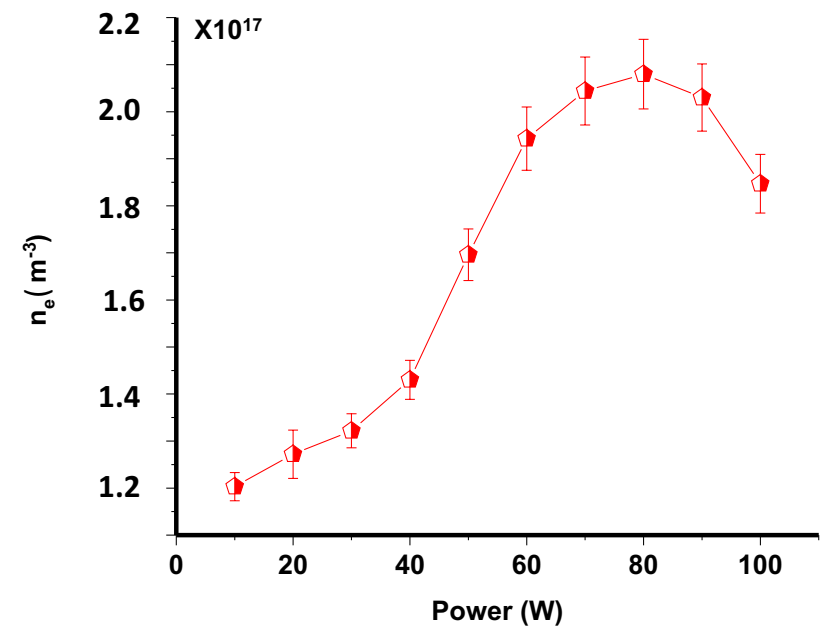

Fig. 7 Electron number density measurements. a The trend of $n_{e}$ with pressure. $\mathbf{b}$ The trend of $n_{e}$ with RF power density with changing RF power when the fill pressure is kept constant. The plot again shows an increase in the number density attributed to the electron heating at higher RF powers that result in a higher ionization rate. Actually, with increasing RF power, the electron impact ionization process produces more ions and electrons and therefore increases the electron number density. However, in this case, the electron temperature also increases owing to compensation of energy loss of electrons involving in the collisional process due to their continuous RF heating.

\section{Conclusions}

In this work, capacitively coupled radio frequency nitrogen plasma at a fixed $13.6 \mathrm{MHz}$ frequency has been generated and subsequently investigated for plasma parameters using optical emission spectroscopy. The emission intensity of the selected spectral lines/bands has been analyzed to evaluate the vibrational temperature $T_{v^{\prime}}$, electron temperature $T_{e^{\prime}}$ and electron number density $n_{e}$ at different nitrogen fill pressure ( 0.1 to $1 \mathrm{mbar}$ ) and radio frequency power (10-100 W) by keeping the other corresponding parameters fixed. First, we used the population density $N_{2}\left(C^{3} \Pi_{u}, v^{\prime}\right)$ of the second positive system $N_{2}\left(C, v^{\prime}-B, v^{\prime \prime}\right)$ for the measurement of vibrational temperature via both the line-ratio and the Boltzmann plot methods. The values of the vibrational temperature obtained from the line-ratio method are slightly higher than the Boltzmann plot method. The difference may be because the line-ratio method considers only the intensity of the two spectral lines and thus represents a narrow segment of the electron energy distribution function contributing to the selected optical transitions. Whereas the Boltzmann plots method uses the intensity of the several spectral lines instead of two lines and thus represents a broadened segment of the electron energy distribution function taking part in the optical transitions. The ratio of the intensity of nitrogen molecular ion $N_{2}^{+}(B, 0-X, 0)$ at $391.44 \mathrm{~nm}$ and molecule $\mathrm{N}_{2}(C, 0-B, 0)$ at $375.4 \mathrm{~nm}$ has been taken into account for the quantification of $T_{e}$. For $n_{e}$ the intensity ratio of nitrogen ion $N_{2}^{+}(B, 0-X, 0)$ at $391.44 \mathrm{~nm}$ and nitrogen molecule $\mathrm{N}_{2}(C, 0-B, 2)$ at $380.5 \mathrm{~nm}$ has been used along with spectroscopic constants. The results show that with increasing radio frequency power both $n_{e}$ and $T_{e}$ increases but $T_{v}$ reduces. The increase in $T_{e}$ and decrease in $T_{v}$ with increasing RF power may be explained by the fact that electronic excitation is more sensitive to highenergy electrons compared with vibrational excitation. At the higher value of RF power, the EEDF shifts to higher energies reducing the number of low energy electrons responsible for the vibrational excitations. Consequently, the vibrational temperature decreases relative to electron 
temperature. On the other hand, with increasing nitrogen fill pressure $n_{e}$ increases but both $T_{v}$ and $T_{e}$ decrease.

Acknowledgements The authors thank the team of the Pakistan TOKAMAK Plasma Research Institute for their cooperation and support. The authors declare that accepted principles of ethical and professional conduct have been followed in this research. Moreover, there are no potential conflicts of interest (financial or non-financial) and the research involves the human participants with consent and does not involve animals.

Funding The authors acknowledge the financial support from the Planning Commission of Pakistan and IAEA through Coordinated Research Project (CRP) "Utilization of the Network of Small and Medium Size Magnetic Confinement Fusion Devices for Fusion Research (F13019)" under Contract No. 22771.

\section{Declarations}

Conflict of interest The authors declare that they have no known competing financial interests or personal relationships that could have appeared to influence the work reported in this paper.

Open Access This article is licensed under a Creative Commons Attribution 4.0 International License, which permits use, sharing, adaptation, distribution and reproduction in any medium or format, as long as you give appropriate credit to the original author(s) and the source, provide a link to the Creative Commons licence, and indicate if changes were made. The images or other third party material in this article are included in the article's Creative Commons licence, unless indicated otherwise in a credit line to the material. If material is not included in the article's Creative Commons licence and your intended use is not permitted by statutory regulation or exceeds the permitted use, you will need to obtain permission directly from the copyright holder. To view a copy of this licence, visit http://creativecommons. org/licenses/by/4.0/.

\section{References}

1. Sung YM, Kim HJ (2007) Sputter deposition and surface treatment of TiO2 films for dye-sensitized solar cells using reactive RF plasma. Thin Solid Films 12:4996

2. Qayyum A et al (2021) Swept Langmuir probe investigation of a time varying DC discharge SN. Appl Sci 3(1):1-10

3. Gupta B et al (2000) Surface modification of polyester films by RF plasma. J Appl Polym Sci 78:1083

4. Liu CZ et al (2004) Comparative study on the effect of RF and DBD plasma treatment on PTFE surface modification. Mater Chem Phys 85:340

5. Chu PK et al (2002) Plasma-surface modification of biomaterials. Mater Sci Eng R Rep 36:143

6. Qayyum A et al (2007) Glow discharge plasma nitriding of AISI 304 stainless steel. Plasma Sci Technol 9:463

7. Nixon DE, Velmer AF, Richard NK (1974) Inductively coupled plasma-optical emission analytical spectroscopy. Tantalum filament vaporization of microliter samples. Anal Chem 46:210

8. Pai PG et al (1986) Infrared spectroscopic study of SiO x films produced by plasma enhanced chemical vapor deposition. JVac Sci Technol A 4:689
9. Laux CO et al (2003) Optical diagnostics of atmospheric pressure air plasmas. Plasma Sources Sci and Technol 12:125

10. Biloiu C et al (2007) An alternative method for gas temperature determination in nitrogen plasmas: Fits of the bands of the first positive system (B П $3 \mathrm{~g} \rightarrow \mathrm{A} \Sigma 3 \mathrm{u}+$ ). J Appl Phys 101:073303

11. Qayyum A et al (2007) Optical emission spectroscopy of $\mathrm{Ar}-\mathrm{N} 2$ mixture plasma. J Quant Spectrosc Radiat Transfer 107:361

12. Yuan $Q$ et al (2020) The optical emission spectroscopy of nitrogen plasma driven by the $94.92 \mathrm{MHz} / 13.56 \mathrm{MHz}$ dual-frequency. Phys Lett A 384:126367

13. Levaton J et al (2004) A detailed discussion of the $\mathrm{N} 2$ (C 3Пu) and $\mathrm{N} 2(\mathrm{X} 1 \Sigma \mathrm{g}+)$ vibrational temperatures in N2 glow discharges. Eur Phys J Appl Phys 26:59

14. Qayyum A et al (2020) Optical actinometric measurements of nitrogen impurity in Ar/He microwave discharge during wall cleaning of MT-I spherical tokamak. Vacuum 182:109672

15. Yun Wu et al (2010) Experimental investigation into characteristics of plasma aerodynamic actuation generated by dielectric barrier discharge. Chin J Aeronaut 23:39

16. Bayram SB, Freamat MV (2012) Vibrational spectra of N2: An advanced undergraduate laboratory in atomic and molecular spectroscopy. Am J of Phys 80:664

17. Qayyum A et al (2010) Vibrational Distribution of N2 (C, v) State in a Pulsed-DC Generated N2-Ar Glow Discharge. Spectrosc Lett 43:259

18. Isola LM, Gómez BJ, Vasco G (2009) Determination of the electron temperature and density in the negative glow of a nitrogen pulsed discharge using optical emission spectroscopy. J Phys D Appl Phys 4:015202

19. Dyatko NA, Kochetov IV, Napartovich AP (2002) Electron temperature in nitrogen afterglow: Dependence of theoretical results on the adopted set of cross sections and on the type of molecular distribution over vibrational levels. Plasma Phys Rep 28:965

20. Zhu XM, Pu YK (2006) A molecular kinetic model for the optical emission spectroscopy technique in inductively coupled nitrogen plasma. Phys Plasmas 13:063507

21. Guerra V, Sá PA, Loureiro J (2004) Kinetic modeling of low-pressure nitrogen discharges and post-discharges. Eur Phys J Appl Phys 28:125

22. Brühl SP et al (1997) A study by emission spectroscopy of the active species in pulsed DC discharges. J Phys D Appl Phys 30:2917

23. Annušová A et al (2012) Spectroscopic diagnostics and modelling of a N2-Ar mixture discharge created by an RF helical coupling device: II. Vibrational distribution of the N2 (C 3Пu, v') state. Plasma Sources Sci Technol 21:055022

24. Hartmann G, Johnson PC (1978) Measurements of relative transition probabilities and the variation of the electronic transition moment for N2 C3Пu-B3 $\Pi g$ second positive system. J Phys B Atom Mol Phys 11:1597

25. Angus WR (1941) The identification of molecular spectra. Nature $148: 240$

26. Naveed MA et al (2006) Effects of helium gas mixing on the production of active species in nitrogen plasma. Phys Lett $A$ 359:499-503

27. Drake DJ, Popović S, Vušković L (2008) Characterization of a supersonic microwave discharge in $\mathrm{Ar} / \mathrm{H}_{2} /$ Air mixtures. J Appl Phys 104:063305

28. Qayyum A et al (2005) Diagnostics of nitrogen plasma by trace rare-gas-optical emission spectroscopy. J Appl Phys 98:103303 
29. Mallick C, Bandyopadhyay M, Kumar R (2018) Plasma characterization of a microwave discharge ion source with mirror magnetic field configuration. Rev Sci Instrum 89:125112

30. Qayyum A et al (2019) Optical emission and langmuir probe diagnostic measurements in DC electrode pulse discharge in nitrogen. High Temp 57:821
Publisher's Note Springer Nature remains neutral with regard to jurisdictional claims in published maps and institutional affiliations. 